\title{
A SEMI-INFINITE QUADRATIC PROGRAMMING ALGORITHM WITH APPLICATIONS TO CHANNEL EQUALIZATION
}

\author{
To Tran, Mattias Dahl, and Ingvar Claesson \\ Blekinge Institute of Technology \\ Department of Telecommunications and Signal Processing \\ Box 520, SE-372 25 Ronneby, Sweden
}

\begin{abstract}
This paper is based on DNCA (Dual Nested Complex Approximation) for optimizing communication channel equalizers using semi-infinite quadratic programming. The optimality criterion for the equalizer is either to minimize the complex deviation in the passband or to minimize its stopband energy when subjected to a specified peak side lobe level in the stopband. Additional linear constraints can be used to form the response by means of group delay, nulls etc. The design approach is applied to a numerical example which deals with the design of a complex communication channel equalizer.
\end{abstract}

\section{INTRODUCTION}

In signal processing applications, the design of digital filters are governed by two basic norm criteria: the $\mathcal{L}_{2}$-norm (least squares) criterion and the $\mathcal{L}_{\infty}$-norm (minimax) criterion, cf. e.g. [1]-[8]. These two criteria are commonly related to noise gain and magnitude response specifications, respectively. However, the most flexible design approach in a practical situation is to consider the combination of these methods, i.e. the tradeoff between the least squares and the minimax errors.

A new optimal window was defined in [9], providing the optimum tradeoff between the peak side lobe level and the stopband energy for FIR filters. It was demonstrated that the classical minimax and least squares methods (Dolph-Chebyshev/prolatespheriodal windows) are both fundamentally inefficient with respect to the other design criterion (the minimax window has the highest stopband energy etc.).

The optimum window [9] minimizes the stopband energy subjected to a specified peak side lobe level together with some additional linear constraints in the passband. The solution for linearphase FIR filters (symmetric windows) can be found by conventional quadratic programming methods [10] since the magnituderesponse can be represented by a real amplitude function [4].

In this paper, we extend the definition in [9], and consider the design of general digital equalizer with complex response [1]. The performance measure used here is either the deviation in the passband of the equalizer [11] or its stopband energy, both quadratic functions of the filter weights. The design criterion is to optimize the performance when subjected to a specified peak side lobe level and a desired complex response in the passband. By employing the real rotation theorem [4], the optimum equalizing filter design is formulated as a semi-infinite quadratic program. A new complex approximation method called DNCA (Dual Nested Complex Approximation) is used to obtain the solution of the optimization problem, cf. [12].
We emphasize that the DNCA design technique may be useful for complex approximation with any filter having linear structure such as the digital Laguerre networks [13], narrowband as well as broadband beamformers $[1,14,15]$ and digital FIR equalizers [6]. Thus, the semi-infinite quadratic programming technique may be used as a means for interpolation between the $\mathcal{L}_{2}-$ and $\mathcal{L}_{\infty}$-norms when the filter response is complex, the corresponding basis is finite and the specification is given in terms of amplitude and phase. Furthermore, the DNCA optimization approach is advantageous due to its flexibility with respect to additional specifications in frequency, time and space (group delay, envelope constraints, null constraints, etc.), cf. [16].

A number of numerical methods exist for the solution of general semi-infinite programming problems, see e.g. [17, 18, 19]. However, most methods are computationally demanding and employ approximation by discretization in some step of the optimization procedure. The DNCA is a simple and numerically efficient technique exploiting a result from Caratheodory dimensionality theory to avoid the need of discretization [20]. The advantages of this procedure are: the constraint set can be represented in functional form rather than stored in memory as numerical values; a finite active set having (at most) the same size as the number of filter parameters need to be considered at any one step in the optimization process; the algorithm deals directly with the true complex error and not an approximation thereof; the design technique is directly applicable to a situation where measured responses are available.

The rest of the paper is organized as follows: In section II, the communication channel equalization problem is formulated as a semi-infinite quadratic program, where the total channel response must be considered in the complex domain. Section III presents the main idea in the DNCA-QP optimization algorithm. In section IV a numerical example concerning the design of a digital equalizer $h(n)$ with complex response using the new optimization strategy DNCA is considered. Section V concludes the paper.

\section{PROBLEM FORMULATION}

Total Transmisssion Channel $\quad \mathrm{T}(\omega)$

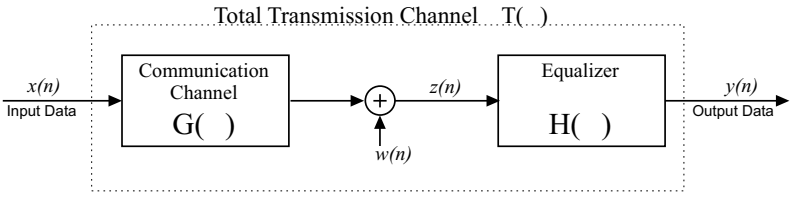

Fig. 1.

As an example of temporal signal processing problem, con- 
sider the design of an equalizer for a communication channel illustrated in figure 1 above. The communication channel $G(\omega)$ with the unknown noise source corrupts the input signal $x(n)$. The digital equalizer $H(\omega)$ with complex response is used to reduced or eliminated the distortion introduced. The desired complex response for the total transmission channel $T_{d}(\omega)$ contains both amplitude and phase constraints. The goal is to equalize the channel $T(\omega)=G(\omega) H(\omega)$, which should be equivalent to the desired response in the passband $T_{d}(\omega) \equiv T(\omega)$. Normally, due to the nature of an actual transmission channel, this goal is in practice not possible to reach, only an approximation thereof. Since the noise is unknown, the stopband energy is minimized. The desired response $T_{d}(\omega)$ for the equalized channel consists of a passband and a stopband, with a transition region in between. The specification for the passband includes both amplitude and phase demands while the stopband specification is done only in the amplitude domain. The phase specification corresponds to a group delay equalization of the channel in the passband. The amplitude in the stopband region should be as small as possible under the fulfilment of the stipulated passband requirements.

The frequency response $H(\omega)$ of the equalizer $h(n)$ can be written as

$$
H(\omega)=\sum_{n=0}^{N-1} h(n) e^{-j \omega n}=\phi^{T}(\omega) \mathbf{h}
$$

where $N$ is the filter length. The response can be expressed more compactly using vector notation where

$\phi(\omega)=\left[1, e^{-j \omega}, \ldots, e^{-j \omega(N-1)}\right]^{T}$ is the response vector and $\mathbf{h}=[h(0), h(1), \ldots, h(N-1)]^{T}$ denotes the filter vector.

The design problem is to minimize the energy $E_{s}$ in the stopband $\Omega_{s} \in\left[\omega_{s}, \pi\right]$ and is defined as

$$
E_{s}=\frac{1}{\pi} \int_{\omega_{s}}^{\pi}|T(\omega)|^{2} d \omega=\frac{1}{\pi} \int_{\omega_{s}}^{\pi}\left|G(\omega) \phi^{T}(\omega) \mathbf{h}\right|^{2} d \omega
$$

while additionally satisfying the constraints in the passband with a tolerance $\sigma(\omega)$. The problem is formulated as

$$
\begin{aligned}
& \min E_{s}, \quad \text { subject to } \\
& \left|G(\omega) H(\omega)-T_{d}(\omega)\right| \leq \sigma(\omega), \quad \omega \in \Omega_{p} .
\end{aligned}
$$

The constraints is defined only in the passband region $\Omega_{p} \in\left[0, \omega_{p}\right]$, but can easily be extended to include constraints in the stopband, such as peak side lobe level.

According to the real rotation theorem [4], a magnitude inequality in the complex plane can be expressed in the equivalent form

$$
|z| \leq \sigma \quad \Leftrightarrow \quad \Re\left\{z e^{j \theta}\right\} \leq \sigma \quad \forall \theta \in[0,2 \pi]
$$

where $\Re\{\cdot\}$ denotes the real part of $\{\cdot\}$ and the phase $\theta$ belongs to the infinite set $\Theta=[0,2 \pi]$.

By making use of (4), the design problem (3) can be formulated as:

$$
\left\{\begin{array}{l}
\min \frac{1}{2} \mathbf{h}^{T} \mathbf{R h} \\
\mathbf{a}(\omega, \theta) \leq \sigma(\omega), \forall \theta \in \Theta, \omega \in \Omega_{p}
\end{array}\right.
$$

where

$$
\mathbf{a}(\omega, \theta)=\Re\left\{\left(G(\omega) \phi^{T}(\omega) \mathbf{h}-T_{d}(\omega)\right) e^{j \theta}\right\} .
$$

In a discrete frequency domain $\left(\omega_{i} \in\left[0, \omega_{p}\right], i=1, \ldots, I\right)$, the design problem can now be formulated as a semi-infinite quadratic program

$$
\left\{\begin{array}{l}
\min \frac{1}{2} \mathbf{h}^{T} \mathbf{R h} \\
\mathbf{a}\left(\omega_{i}, \theta\right) \leq \sigma\left(\omega_{i}\right) \quad \forall \theta \in \Theta, \quad \omega_{i} \in \Omega_{p} .
\end{array}\right.
$$

The matrix $\mathbf{R}$ in the objective function can be defined in two ways: either to minimize the stopband energy in the transmission channel $T(\omega)$ or the stopband energy for the equalizer $H(\omega)$. When minimizing the transmission channel energy, the matrix is defined as

$$
\mathbf{R}=\Re\left\{\frac{1}{\pi} \int_{\omega_{s}}^{\pi}|G(\omega)|^{2} \phi(\omega) \phi^{H}(\omega) d \omega\right\}
$$

and in the second case, where the equalizer energy is minimized,

$$
\mathbf{R}=\Re\left\{\frac{1}{\pi} \int_{\omega_{s}}^{\pi} \phi(\omega) \phi^{H}(\omega) d \omega\right\}
$$

The quadratic program (7), solving a non-linear program like this is called semi-infinite since the number of variables (unknowns) are finite but the constraint set is infinite. For practical purposes in the algorithm implementation, it is assumed that the set $\omega_{i}$ is finite. Note that the corresponding approximation problem is with respect to the true complex error since the phase variable $\theta$ belongs to an infinite set.

The problem formulated in (7) can be solved by using the new DNCA semi-infinite programming algorithm.

\section{THE DNCA-QP OPTIMIZATION ALGORITHM}

Consider the semi-infinite optimization problem

$$
(P)\left\{\begin{array}{l}
\min _{\mathbf{h}} f(\mathbf{h}), \\
g_{\alpha}(\mathbf{h}) \leq 0, \alpha \in \mathcal{A} \subset R^{k} \\
\mathbf{h} \in \mathcal{H} \subset R^{N}
\end{array}\right.
$$

where $\mathbf{h}$ is an $N \times 1$ variable vector, $f(\mathbf{h})$ a convex continuous function, $\mathcal{H}$ a convex restriction set as a compact subset of Euclidean $N$-space, $\mathcal{A}$ an infinite index set as a compact subset of Euclidean $k$-space $\left(R^{k}\right)$, and $g_{\alpha}(\mathbf{h})$ a continuous constraint function which is convex for any fixed index $\alpha$.

The Dual Nested Complex Approximation Quadratic Programming (DNCA-QP) algorithm to solve (10) is outlined below. Let $\mathcal{A}^{(k)}$ denote a sequence of finite subsets of the infinite index set $\mathcal{A}$ and initialize the algorithm with the subset $\mathcal{A}^{(0)}$.

1. Given $\mathcal{A}^{(k)} \subset \mathcal{A}$, solve the subproblem

$$
\left(P^{(k)}\right)\left\{\begin{array}{l}
\min _{\mathbf{h}} f(\mathbf{h}), \mathbf{h} \in \mathcal{H} \\
g_{\alpha_{i}}(\mathbf{h}) \leq 0, \alpha_{i} \in \mathcal{A}^{(k)}
\end{array}\right.
$$

yielding the solution vector $\mathbf{h}_{k}$ and the Lagrange multiplier vector $\boldsymbol{\lambda}_{k}$.

2. Reduce the subset by the inactive constraints

3. Define the entering index

$$
\mathcal{A}_{R}^{(k)}=\mathcal{A}^{(k)} \backslash\left\{\alpha_{i} \in \mathcal{A}^{(k)} \mid\left(\boldsymbol{\lambda}_{k}\right)_{i}=0\right\}
$$

$$
\begin{aligned}
\hat{\alpha}_{k} & =\arg \max _{\alpha} g_{\alpha}\left(\mathbf{h}_{k}\right), \\
\mathcal{A}^{(k+1)} & =\mathcal{A}_{R}^{(k)} \cup\left\{\hat{\alpha}_{k}\right\}
\end{aligned}
$$

and return to step 1 above. The iteration stops when

$g_{\alpha}(\mathbf{h})<0+\varepsilon$, where $\varepsilon$ is a predefined tolerance parameter.

Since the reduced subset $\mathcal{A}_{r}^{(k)}$ yields the same solution $\mathbf{h}_{k}$ as the subset $\mathcal{A}^{(k)}$ we have $f\left(\mathbf{h}_{k}\right) \leq f\left(\mathbf{h}_{k+1}\right) \leq f_{\text {opt }}$ and the sequence $f\left(\mathbf{h}_{k}\right)$ converges. However, it remains to be shown that the sequence $\mathbf{h}_{k}$ is not locked in any state of cycling.

Convergence can be proved straightforwardly when the cost function $f(\mathbf{h})$ is strictly convex. Assume that $\mathbf{h}_{k}$ is not optimal, then $g_{\hat{\alpha}}\left(\mathbf{h}_{k}\right)>0$. The claim is that there is a strict ascent, 
$f\left(\mathbf{h}_{k+1}\right)>f\left(\mathbf{h}_{k}\right)$, so that cycling cannot occur. Assume on the contrary that $f\left(\mathbf{h}_{k+1}\right) \leq f\left(\mathbf{h}_{k}\right)$. Define

$\Omega_{k}=\left\{\mathbf{h} \mid g_{\alpha}(\mathbf{h}) \leq 0, \alpha \in \mathcal{A}^{(k)}\right\}$ and $\Omega_{k+1}=\Omega_{k} \cap\left\{\mathbf{h} \mid g_{\hat{\alpha}}(\mathbf{h}) \leq 0\right\}$.

Obviously $\mathbf{h}_{k} \in \Omega_{k}, \mathbf{h}_{k+1} \in \Omega_{k}, \mathbf{h}_{k+1} \in \Omega_{k+1}$ and $\mathbf{h}_{k} \notin \Omega_{k+1}$.

Thus, $\mathbf{h}_{k} \neq \mathbf{h}_{k+1}$ and $f\left(t \mathbf{h}_{k}+(1-t) \mathbf{h}_{k+1}\right)<t f\left(\mathbf{h}_{k}\right)+(1-$ t) $f\left(\mathbf{h}_{k+1}\right) \leq t f\left(\mathbf{h}_{k}\right)+(1-t) f\left(\mathbf{h}_{k}\right)=f\left(\mathbf{h}_{k}\right)$ for any $0<t<1$ which contradicts the fact that $\mathbf{h}_{k}$ is optimum on $\Omega_{k}$.

If the cost function $f(\mathbf{h})$ is not strictly convex, strict ascent can still be obtained by requiring that the condition $f\left(\mathbf{h}_{k}\right)>f\left(\mathbf{h}_{k-1}\right)$ be satisfied in order to execute step 2 (Eq. (12)) in the DNCA algorithm.

It is important to note that each subproblem requires no more than $N$ linear constraints according to the finite dimensionality property of the related Lagrange multipliers. This section is concluded be these three key observations:

Key observation 1 Since the number of variables are $N$, we note that an optimization software will usually give us a total of $N$ lagrange multipliers greater than zero. The size of the so called reference set $\mathcal{A}_{R}^{(k)}$ is in fact $r \leq N$.

Key observation 2 The dual formulation of primal problem in (7) can be solved by considering a sequence of subproblems of the dual formulation with increasing minimum cost and which is based only on finite subsets $\mathcal{A}_{R}^{(k)}$ consisting of no more than $N$ points of $\mathcal{D}=\Omega_{p} \times \Theta$. This observation constitutes the foundation for the development of the DNCA-QP optimization algorithm.

Key observation 3 The number of variables is $N$ and the size of the reduced reference set $\mathcal{A}_{R}^{(k)}$ is only $r \leq N$. The entering constraint $\hat{\alpha}_{k}$ which is chosen to enter the basis $\mathcal{A}_{R}^{(k)}$ is usually defined by the maximum constraint violation. Hence, this entering constraint $\hat{\alpha}_{k}$ is very likely to be independent of the small reduced reference set $\mathcal{A}_{R}^{(k)}$. This is the primary reason ensuring that the DNCA itself is a highly numerical stable procedure.

\section{NUMERICAL EXAMPLE}

Consider the equalization of a transmission channel $G(\omega)$ given by a 4th order lowpass digital elliptic (Cauer) IIR filter with $0.5 \mathrm{~dB}$ ripple in the passband, a stopband attenuation of $34 \mathrm{~dB}$, and cut-off frequency $\omega_{c}=\frac{\pi}{2}$.

The desired total response is defined as

$$
T_{d}(\omega)= \begin{cases}e^{-j \omega \tau} & \omega \in \Omega_{p}=\left[0, \omega_{p}\right], \omega_{p}=\frac{\pi}{2} \\ 0 & \omega \in \Omega_{s}=\left[\omega_{s}, \pi\right], \omega_{s}=\frac{3 \pi}{5}\end{cases}
$$

where $\tau=13$, which corresponds to a groupdelay of 13 samples. Consider the design of a $N=31$ taps digital FIR equalizer $h(n)$ with complex response, as described in section II. The semiinfinite quadratic program for this design example is given by:

$$
\left\{\begin{array}{l}
\min \frac{1}{2} \mathbf{h}^{T} \mathbf{R h} \\
\mathbf{a}_{\tau}\left(\omega_{i}, \theta\right) \leq \sigma\left(\omega_{i}\right), \forall \theta \in[0,2 \pi], \omega_{i} \in \Omega_{p}
\end{array}\right.
$$

where

$$
\left.\mathbf{a}_{\tau}\left(\omega_{i}, \theta\right)=\Re\left\{\left(G\left(\omega_{i}\right) \phi^{T}\left(\omega_{i}\right) \mathbf{h}-e^{-j \omega \tau}\right) e^{j \theta}\right)\right\}
$$

Note that the objective function is defined only in the stopband. The maximum allowed passband ripple is set to $0.1 \mathrm{~dB}$, which implies $\sigma\left(\omega_{i}\right) \approx 0.01157$ for $\omega_{i} \in \Omega_{p}$. As mentioned in section II, the objective function can be formulated to either minimize the stopband energy for equalizer (case 1) or to minimize the stopband energy for the total transmission channel (case 2). However, the
DNCA-QP algorithm can be used to solve both cases. When minimizing the energy of the equalizer, the matrix $\mathbf{R}$ can be expressed explicitly as:

$$
R_{n m}=\left\{\begin{array}{cc}
\frac{\pi-\omega_{s}}{\pi} & m=n \\
-\frac{\sin \left(\omega_{s}(n-m)\right)}{\pi(n-m)} & m \neq n
\end{array}\right.
$$

where $R_{n m}$ are the elements of $\mathbf{R}$.

If the channel $G(\omega)$ is taken under consideration then the matrix $\mathbf{R}$ becomes

$$
\mathbf{R}=\frac{\pi-\omega_{s}}{I \pi} \Re\left\{\Phi \mathbf{W} \Phi^{H}\right\}
$$

where $\mathbf{W}$ is a diagonal matrix with the diagonal elements $w_{i}$ defined as $w_{i}=\left|G\left(\omega_{i}\right)\right|^{2}$.

Figure 2 shows the response of equalizer $H(\omega)$ for both cases. Figure 3 shows the equalized channel (total transmission channel) $T(\omega)=G(\omega) H(\omega)$. Figure 4 shows the group delay of the channel before and after equalization for both cases. Note the difference between the equalizer responses for the two cases, while the group delay is almost the same. In this design example, the algorithm converges in less than 100 iterations.

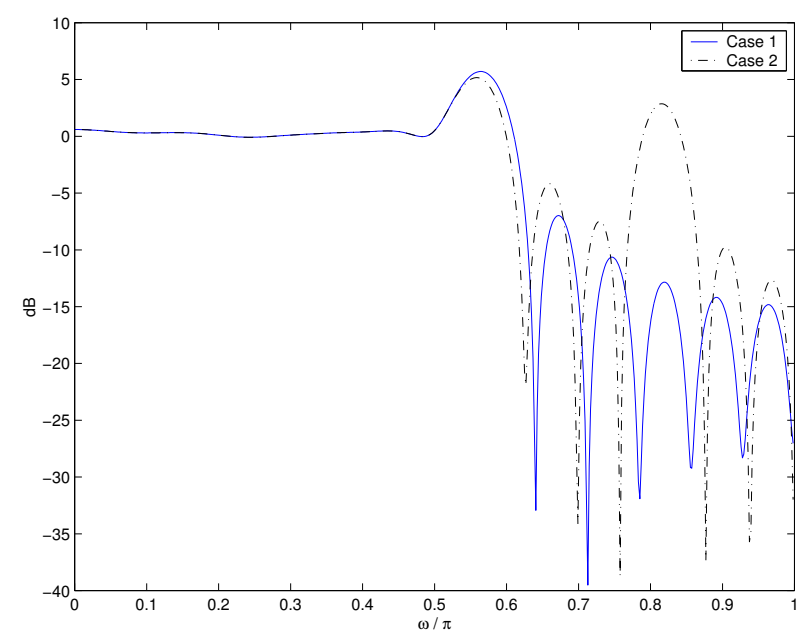

Fig. 2. Equalizer response for both case 1 and case 2. Note the difference between the equalizer response for the two cases.

\section{CONCLUSIONS}

This paper demonstrates the advantages gained by using the DNCA algorithm for optimizing equalizers by semi-infinite quadratic programming. The advantages of this procedure are: The constraint set can be represented as functional form rather than stored in memory as numerical values; a finite active set having (at most) the same size as the number of filter parameters need to be considered at any one step in the optimization process; the algorithm deals directly with the true complex error and not an approximation thereof; the design technique is directly applicable to a situation where measured responses are available. We have also presented two different design approaches, either minimizing the stopband energy for the total transmission channel or to minimize the stopband energy for the equalizer. 


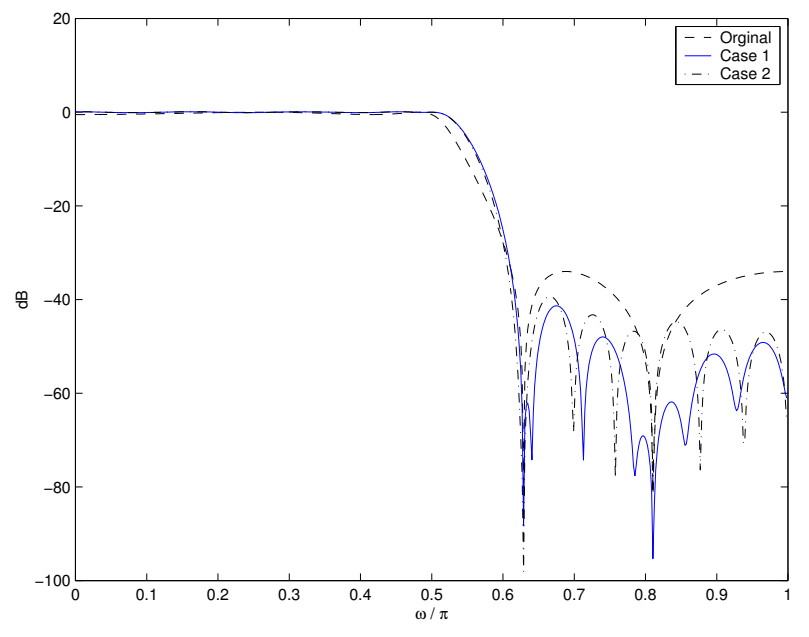

Fig. 3. The original channel response before equalization and after equalization for both case 1 and case 2 .

\section{REFERENCES}

[1] B. D. Van Veen, K. M. Buckley, "Beamforming: A Versatile Approach to Spatial Filtering", IEEE ASSP Magazine, pp. 4-24, April 1988.

[2] R. L. Streit, A. H. Nuttall, "A general Chebyshev complex function approximation procedure and an application to beamforming", Journal of the Acoustical Society of America, vol. 72, no. 1, pp. 181-190, July 1982.

[3] R. L. Streit, A. H. Nuttall, "A Note on the Semi-Infinite Programming Approach to Complex Approximation", Mathematics of Computation, vol. 40, no. 162, pp. 599-605, April 1983.

[4] T. W. Parks, C. S. Burrus, Digital Filter Design, John Wiley \& Sons, Inc., 1987.

[5] X. Chen, T. W. Parks, "Design of FIR Filters in the Complex Domain", IEEE Transactions on Acoustics, Speech, and Signal Processing, vol. ASSP-35, no. 2, pp. 144-153, February 1987.

[6] K. Preuss, "On the Design of FIR Filters by Complex Chebyshev Approximation", IEEE Transactions on Acoustics, Speech, and Signal Processing, vol. 37, no. 5, pp. 702712, May 1989.

[7] A. S. Alkhairy, K. G. Christian, J. S. Lim, "Design and Characterization of Optimal FIR Filters with Arbitrary Phase" IEEE Transactions on Signal Processing, vol. 41, no. 2, pp. 559-572, February 1993.

[8] D. Burnside, T. W. Parks, "Optimal Design of FIR Filters with the Complex Chebyshev Error Criteria", IEEE Transactions on Signal Processing, vol. 43, no. 3, pp. 605-616, March 1995.

[9] J. W. Adams, "A new optimal window", IEEE Trans. Signal Processing, vol. 39, no. 8, pp. 1753-1769, August 1991.

[10] D. G. Luenberger, Linear and Nonlinear Programming, Addison-Wesley, 1984.

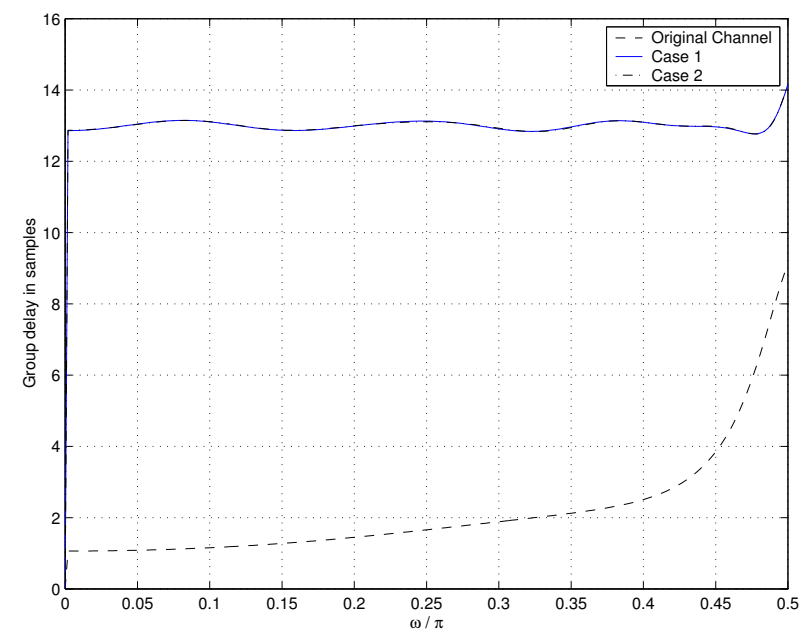

Fig. 4. The group delay of the original channel and the equalized channels for both cases. The group delay for case 1 and 2 is almost equal.

[11] D. K. Cheng, "Field and Wave Electromagnetics", pp. 610612, Addison-Wesley 1989.

[12] M. Dahl, S. Nordebo, I. Claesson, "Complex Approximation by Semi-Infinite Quadratic Programming", in Proc. of ISPACS2000, Honolulu, Hawaii, U.

[13] B. Wahlberg, "System Identification Using Laguerre Models", IEEE Transactions on Automatic Control, vol. 36, no. 5, pp. 551-562, May 1991.

[14] S. Nordebo, I. Claesson, S. Nordholm, "Weighted Chebyshev Approximation for the Design of Broadband Beamformers Using Quadratic Programming", IEEE Signal Processing Letters, vol. 1, no. 7, pp. 103-105, July 1994.

[15] S. Nordholm, V. Rehbock, K. L. Teo, S. Nordebo, "Chebyshev Optimization for the Design of Broadband Beamformers in the Near Field", IEEE Transactions on Circuits and Systems II, vol. 45, no. 1, pp. 141-144, January 1998.

[16] S. Nordebo, Z. Zang, "Semi-Infinite Linear Programming: A Unified Approach to Digital Filter Design with Time and Frequency Domain Specifications", IEEE Transactions on Circuits and Systems-II: Analog and Digital Signal Processing, vol. 46, no. 6, June 1999.

[17] H. Lebret, S. Boyd, "Antenna Array Pattern Synthesis via Convex Optimization”, IEEE Transactions on Signal Processing, vol. 45, no. 3, pp. 526-532, March 1997.

[18] R. Hettich, K. O. Kortanek, "Semi-Infinite Programming: Theory, Methods, and Applications", SIAM Review, vol. 35, No. 3, pp. 380-429, September 1993.

[19] R. Hettich, "A Review of Numerical Methods for SemiInfinite Programming and Applications". In A. V. Fiacco, K. O. Kortanek, Editors, Semi-Infinite Programming and Applications, pp. 158-178, Springer-Verlag, Berlin, 1983. S. A., November 2000. vol. II, pp. 876-881.

[20] S. Nordebo, I. Claesson, Z. Zang, "Optimum Window Design by Semi-Infinite Quadratic Programming”, IEEE Signal Processing Letters, vol. 6, no. 10, October 1999. 